\title{
Original
}

\section{Resección esofágica por cáncer, experiencia del Servicio de Cirugía de Tórax del Hospital Dr. Rafael Á. Calderón Guardia}

\author{
José Alberto Mainieri-Hidalgo
}

\section{Resumen}

Durante el período comprendido entre 1990 y 2006, 28 pacientes fueron sometidos a resección esofágica por cáncer en el Servicio de Cirugía de Tórax del Hospital Dr. Rafael Á. Calderón Guardia. La edad promedio fue de 65 años y la relación hombre mujer, de 6 a 1 . El antecedente de tabaquismo se confirmó en un $80 \%$, etilismo en un $75 \%$ y 8 pacientes eran conocidos portadores de esófago de Barret. Disfagia, dolor epigástrico y pérdida de peso fueron los síntomas más frecuentes; siendo la epigastralgia debida a otra patología digestiva coexistente, como reflujo o enfermedad péptica. Los pacientes clasificados como estadio IIb, III y IV recibieron terapia de inducción y aunque en 3 de estos no se encontró tumor residual en la pieza quirúrgica, en 2 casos quedó tumor adherido a la aorta. A 21 pacientes se les realizó esofagectomía total con ascenso gástrico y anastomosis cervical y a 7 esofagectomía parcial con ascenso gástrico al tórax en 5 y 2 esófago-yeyuno anastomosis, una intratorácica y otra abdominal. La complicación más frecuente fue la claudicación respiratoria por bronconeumonía, que se produjo en 7 pacientes y que fue mortal en 3. Dos de estos pacientes presentaron además fuga de anastomosis, una en el cuello y otra en el tórax. A largo plazo la complicación más frecuente fue estrechez de la anastomosis, que en los 7 casos se ha manejado con dilataciones. La mortalidad quirúrgica fue del $10.7 \%$ y en los 3 casos la bronconeumonía fue la causa principal. La supervivencia a 5 y 10 años fue del $31.5 \%$ y el $18 \%$, respectivamente.

Descriptores: Cáncer de esófago, cirugía de esófago, ascenso gástrico

Key words: esophageal cancer, esophageal surgery, gastric pull-up

Recibido: 29 de marzo de 2007

Aceptado: 3 de julio de2007

Servicio de Cirugía de Tórax, Hospital Dr. Rafael Ángel Calderón Guardia, Caja Costarricense de Seguro Social Abreviaturas: UTE, ultrasonido transesofágico;TAC, tomografía axial computarizada; US, ultrasonido.

Correspondencia: José Alberto Mainieri-Hidalgo albertom@racsa.co.cr

ISSN 0001-6002/2007/49/4/210-215 Acta Médica Costarricense, $\mathbb{C} 2007$ Colegio de Médicos y Cirujanos
El cáncer del esófago es una neoplasia relativamente infrecuente en Costa Rica. La tasa de mortalidad por 100.000 habitantes en 2004 fue de 1.38 en hombres y de 0.57 en mujeres. ${ }^{1}$ No obstante, la posibilidad de su existencia debe estar presente siempre que se realice una endoscopía por cualquier causa, porque el diagnóstico temprano de cáncer del esófago se acompaña de un buen chance de curación. ${ }^{2-4}$

La neoplasia se origina generalmente en la mucosa, presentándose como ulceración o estrechez y manifestándose principalmente con disfagia que el paciente suele tolerar por algún tiempo hasta que consulta y se realiza el diagnóstico, muchas veces ya adelgazado, desnutrido y en un estadio avanzado. ${ }^{3,4}$

El esofagograma es el estudio inicial para el diagnóstico diferencial de disfagia; ${ }^{5}$ la esofagoscopía sirve para biopsiar hacer el diagnóstico histológico, y el ultrasonido transesofágico, indispensable para establecer el estadio clínico de la enfermedad y decidir el manejo más adecuado.,3,6,7 
Existen enfermedades que anteceden la neoplasia y que deben ser atendidas o sometidas a control periódico, como son la displasia y el esófago de Barret. ${ }^{8,9}$

El tratamiento curativo para la enfermedad en estadio resecable es la cirugía. En los estadios III y IV la quimioterapia y radioterapia preoperatorias usualmente disminuyen el tamaño del tumor y se aumenta la posibilidad de resección quirúrgica, no así la supervivencia. Esto es importante porque muchos pacientes con la terapia de inducción mejoran considerablemente sus síntomas, lo que puede inducir al error de no proseguir con el tratamiento quirúrgico. ${ }^{10-12}$

Algunas características anatómicas particulares del esófago deben ser tomadas en cuenta para escoger el procedimiento quirúrgico. Este órgano cuenta con una rica red linfática en su pared, que permite la diseminación tumoral a lo largo de éste, por lo que se suele extirpar todo. La resección puede practicarse por toracotomía o transhiatalmente, sin que se haya demostrado diferencia en la morbilidad ni en la supervivencia, pero debe considerarse que cuando el tumor ha superado la capa muscular y debido a que no posee una capa serosa, se adhiere rápidamente a la aorta, al bronquio izquierdo o a las venas pulmonares. ${ }^{3,9,13}$

El objetivo de este trabajo es informar los resultados y la experiencia adquiridos en la cirugía para cáncer del esófago en el Servicio de Cirugía de Tórax del Hospital Dr. Rafael Á. Calderón Guardia, durante el período comprendido entre 1990 y 2007.

\section{Materiales y métodos}

De la base de datos del Servicio de Cirugía de Tórax del Hospital Dr. Rafael Á. Calderón Guardia, se seleccionaron todos los pacientes con diagnóstico de cáncer del esófago. Se revisaron los expedientes de los que tuvieron resección esofágica y se analizaron los datos clínicos de laboratorio y gabinete, la cirugía y sus complicaciones y la evolución postoperatoria a corto y largo plazos. Se excluyeron 11 casos en los cuales se practicó solo una yeyunostomía o gastrostomía y un caso de toracotomía exploratoria. Los pacientes que no regresaron a consulta fueron llamados telefónicamente a la casa y se indagó sobre la supervivencia. Los casos de carcinoma temprano fueron todos remitidos por el Centro Nacional de Cáncer Gástrico.

\section{Resultados}

A 28 pacientes se les realizó esofagectomía por cáncer del esófago; 24 eran varones y 4 mujeres, para una relación de 6 a 1 . El paciente más joven tenía 38 años y el mayor, 80 , con una edad promedio de 65 años. Doce pacientes (43\%) tuvieron adenocarcinomas y 16 (57\%), carcinoma epidermoide. La localización del tumor fue distal en 15 casos, 12 en el esófago medio y 1 en el proximal; 2 pacientes tuvieron tumor esofagogástrico.

Se encontró el antecedente de tabaquismo en el $80 \%$ y etilismo en el $75 \%$ del grupo. El tiempo promedio entre la aparición de los síntomas y el diagnóstico fue de 3.4 meses. Los síntomas más frecuentes fueron disfagia en $88 \%$ de los casos, dolor epigástrico en $85 \%$, pérdida de peso en $58 \%$, vómito en $44 \%$, tos en $30 \%$ e hipo en $17 \%$. Ocho pacientes tenían el diagnóstico de esófago de Barret previo al de cáncer.

En el 29\% de los casos se detectó anemia; en el 35\%, hipoproteinemia y en $60 \%$, hipoalbuminemia.

El resultado del esofagograma se logró determinar en 9 casos, en 8 de los cuales demostró una estrechez irregular al paso del medio de contraste. La esofagoscopía reportó en 11 casos lesión ulcerada; en 5, estenosis y en 5, lesión vegetante. En 6 pacientes se realizó ultrasonido transesofágico (UTE) que coincidió con la clasificación postoperatoria con 2 diferencias. En un caso que se clasificó como N1, los ganglios fueron reportados como negativos y en un caso en que el UTE lo clasifica como T2, el patólogo encontró un mes y medio después que el tumor abarcaba todas las capas del esófago.

Siete pacientes recibieron quimioterapia preoperatoria y 4 también radioterapia. En 3 de estos casos no se encontró tumor residual en la pieza de patología.

En todos los pacientes incluidos en este estudio el tumor fue extirpado; en 21 casos la resección se consideró completa, en 3 quedó tumor residual macroscópico y en 4 no se define bien este dato. Se realizó esofagectomía total a 21 pacientes: con ascenso gástrico y anastomosis cervical y esofagectomía parcial a 7, con anastomosis torácica en 6 y abdominal en 1 que tenía cáncer esofagogástrico. La esofagectomía en 20 casos se realizó por toracotomía, en 7 por vía transhiatal y en uno solo por laparotomía. El tránsito se reinstauró en 26 casos con ascenso gástrico y en 2 con esófagoyeyunoanastomosis.

El tiempo promedio de duración de la cirugía fue de 5 horas y 20 minutos y el tiempo promedio de estancia postoperatoria fue de 16 días.

Quince pacientes $(53.6 \%)$ tuvieron una o varias complicaciones que se presentan en la Cuadro 1, siendo las más frecuentes bronconeumonía con falla ventilatoria (mortal en 3 casos) y estenosis anastomótica que requirió dilataciones en 6 casos.

El estadio después de revisar los informes de patología se muestra en la Cuadro 2; en un caso no se completaron los datos. 


\begin{tabular}{|l|c|c|}
\hline \multicolumn{3}{|c|}{$\begin{array}{c}\text { Cuadro 1. Complicaciones quirúrgicas en 15 de } \\
28 \text { pacientes operados por cáncer del esófago } \\
\text { en Cirugía de Tórax del Hospital Dr. Rafael Á. } \\
\text { Calderón Guardia }\end{array}$} \\
\hline Complicación & Casos & Porcentaje \\
\hline Bronconeumonía & 7 & $25 \%$ \\
\hline Estenosis & 7 & $25 \%$ \\
\hline Derrame & 3 & $11 \%$ \\
\hline Eventración & 3 & $11 \%$ \\
\hline Sepsis herida & 3 & $11 \%$ \\
\hline Empiema & 2 & $7 \%$ \\
\hline Fuga de anastomosis & 2 & $7 \%$ \\
\hline
\end{tabular}

Cuadro 2. Estadio quirúrgico de 28 pacientes operados por cáncer del esófago

\begin{tabular}{|l|c|}
\hline Estadio & Casos \\
\hline E I & 4 \\
\hline E II a & 14 \\
\hline E II b & 1 \\
\hline E III & 4 \\
\hline E IV a & 3 \\
\hline E IV b & 1 \\
\hline NS & 1 \\
\hline
\end{tabular}

Cuadro 3. Supervivencia de 28 pacientes operados por cáncer del esófago en Cirugía de Tórax del Hospital Dr. Rafael Á. Calderón Guardia

\begin{tabular}{|c|c|c|}
\hline $\begin{array}{c}\text { Años de } \\
\text { supervivencia }\end{array}$ & No. de pacientes & Porcentaje \\
\hline 1 & $9 / 27$ & $33.3 \%$ \\
\hline 2 & $8 / 25$ & $32 \%$ \\
\hline 5 & $6 / 19$ & $31.5 \%$ \\
\hline 10 & $2 / 11$ & $18 \%$ \\
\hline
\end{tabular}

La mortalidad dentro de los 30 primeros días fue de 3 casos, que corresponde a un $10.7 \%$; en todos por bronconeumonía y en 2 hubo además fuga de la anastomosis: una en el cuello y otra en el tórax de una esófagoyeyunoanastomosis. No se encontró relación entre el material de sutura utilizado y la frecuencia de estenosis en los 6 casos, ni en los 2 con fugas anastomóticas.

Se hallaron 27 casos con más de un año de intervenidos y de ellos 9 estaban vivos; 25 tenían más de 2 años de operados y 8 estaban vivos; 19 tenían más de 5 años de operados y 6 estaban vivos, y de los 11 pacientes con más de 10 años, 2 estaban vivos. Cuatro de los pacientes con más de 10 años de operados y 5 con más de 5 años, que se tomaron como fallecidos porque no llegaron a los 10 ó 5 años, respectivamente, murieron por otras causas. (Cuadro 3)

\section{Discusión}

El cáncer del esófago se asocia con condiciones que causan una irritación constante y crónica de la mucosa esofágica como el tabaquismo, la ingesta de bebidas irritantes o muy calientes, la acalasia, el síndrome de Plummer-Vinson, el reflujo gastroesofágico, sobre todo cuando se acompaña de displasia o mucosa de Barret, la mala higiene oral y otros. ${ }^{14-22}$ La displasia de alto grado es considerada de por sí una lesión maligna y por lo tanto se debe tratar como carcinoma in situ..$^{5,23}$ En este estudio se encontró una relación importante con tabaquismo, etilismo y en un $28.6 \%$ de los casos, el cáncer se originó en una mucosa de Barret preexistente.

La enfermedad es propia de adultos mayores, alrededor de los 65 años, y mucho más frecuente en varones, tanto como de 6 a 1, según este estudio., 22,24 Esto, posiblemente, por la mayor frecuencia de factores causantes en los primeros y la tendencia a tolerarlos por más tiempo. En Costa Rica el carcinoma epidermoide (57\%) sigue siendo un poco más frecuente que el adenocarcinoma (43\%).

Es importante señalar que los pacientes con cáncer del esófago suelen haber tenido una o varias endoscopías previas por otros padecimientos digestivos. En este trabajo se encontró que un $85 \%$ de los pacientes tenían antecedente de dolor o malestar epigástrico y varios casos habían sido estudiados previamente con endoscopías.

La disfagia es el síntoma más común y lleva rápidamente a la desnutrición, la cual se manifestó por pérdida de peso en un $58 \%$ de los pacientes e hipoalbuminemia en el $60 \%$. ${ }^{4,9,20,25}$

A pesar de que el esofagograma es el estudio inicial para el diagnóstico diferencial de la disfagia, solo en 9 casos se documentaron sus hallazgos en el expediente, y la endoscopía, que fue en todos los casos el método para tomar la muestra para el diagnóstico histológico, demostró la presencia de ulceración, lesión vegetante o área de estenosis. ${ }^{5,26}$

El UTE es el único estudio que permite valorar la extensión del tumor y establecer el (T) para el estadio clínico. Puede, además, detectar la presencia de adenomegalias periesofágicas (N1) o el compromiso de estructuras adyacentes como aorta, tráquea y venas pulmonares (T4). La disponibilidad de equipo y operador entrenado es fundamental y este estudio faltó en muchos casos de esta serie. Los estudios realizados en el Centro Nacional de Cáncer Gástrico de Cartago coincidieron de manera importante con los hallazgos histológicos. La TAC es útil para detectar metástasis (M1) a pulmón, hígado y ganglios mediastinales. A pesar de que ambos estudios son muy sensibles para detectar adenomegalias, no son específicos, tal como ocurrió en un caso en que los ganglios resultaron ser inflamatorios. 
En presencia de adenomegalias mediastinales o ante la sospecha de invasión de estructuras vitales, se propone el uso de toracoscopía para confirmación histológica. ${ }^{27-29}$ Otros estudios complementarios preoperatorios son; el US de abdomen que busca metástasis a hígado o a ganglios celiacos; la espirometría y valoración de la condición pulmonar; la valoración cardiológica; la determinación de proteínas plasmáticas; la broncoscopía en los tumores del esófago medio, para descartar invasión del bronquio izquierdo; y en los tumores proximales, el US de cuello puede detectar la presencia de adenomegalias cervicales. $3,6,7,26,28$

A los pacientes con estadio I y II se les realizó directamente tratamiento quirúrgico. A 7 pacientes con tumores que llegaban a la capa adventicia (T3) y uno con ganglios periesofágicos (N1), se les aplicó quimioterapia preoperatoria, y a 4 también radioterapia. De estos pacientes con terapia de inducción, en 3 casos no se documentó tumor residual en la pieza quirúrgica. La terapia neoadyuvante ha demostrado ser efectiva para reducir el tamaño del tumor aumentando la posibilidad de resecarlo, pero no disminuye la morbilidad ni aumenta la supervivencia. ${ }^{11,30-32}$

La resección quirúrgica es el único tratamiento que se asocia con curación o con mayor supervivencia. Se ha propuesto la mucosectomía endoscópica, la vaporización con láser o la fulguración con argón para el carcinoma in situ, ${ }^{3,33-35}$ pero el procedimiento estándar es la esofagectomía total, esto porque la diseminación del tumor a lo largo de la pared del órgano es frecuente y porque algunos autores han informado haber encontrado tumores multicéntricos hasta en un $15 \%^{34-36}$, pero sobre todo porque las fugas anastomóticas que son la complicación más temida se manejan mejor en el cuello que en el tórax. ${ }^{37-39}$

Los tumores gastroesofágicos que generalmente son adenocarcinomas originados en el estómago o en el esófago distal en una mucosa de Barret, pueden manejarse con criterio de cáncer gástrico; la esofagectomía distal con margen es apropiada. ${ }^{40}$

La esofagectomía puede realizarse por toracotomía o por vía transhiatal. ${ }^{36,41}$ Salvo para los autores que consideran la disección ganglionar importante, los resultados de supervivencia con ambas son similares, incluyendo la morbilidad y los días de estancia. ${ }^{34,42,43}$ La disección ganglionar es más útil como factor pronóstico que de supervivencia., ${ }^{2,3}$

El tránsito digestivo se puede restaurar con un tubo gástrico, con el colon, y en último caso, con el intestino delgado. ${ }^{43,44}$ En 21 casos del presente estudio se unió el tubo gástrico con un muñón esofágico en el cuello, con una sola fuga anastomótica, pero con varias estenosis que se resolvieron con dilataciones.

La cirugía implica además realizar piloroplastía por el espasmo pilórico que sigue a la vagectomía y yeyunostomía, por la cual se va a nutrir al paciente desde el postoperatorio uno hasta que se reinicie la vía oral, lo que ocurre alrededor de los 10 días de operado y después de confirmar la integridad de la anastomosis radiológicamente, con un trago de bario.

El procedimiento se asocia con un alto grado de complicaciones dentro las cuales y tal como ocurrió con los casos de este estudio, la claudicación respiratoria es la más frecuente, hecho que coincide con la bibliografía revisada, y la fuga anastomótica, la más temida. ${ }^{24,44,45}$ Esta complicación se presentó en 2 casos (7\%), y se reporta en la bibliografía entre un $4 \%$ y un $19 \%{ }^{10,21,43,46}$ En esta serie, los 3 pacientes que fallecieron en el postoperatorio tuvieron neumonía y de ellos 2 también sufrieron fuga de la anastomosis. La fuga en el tórax se consideró la causa de la fatalidad en 1, no así la del cuello, que solamente contribuyó a la neumonía como causa de muerte, ya que se encontraba bien conducida.

La supervivencia a corto plazo depende de las complicaciones operatorias y de la adaptación del paciente a su nuevo estatus fisiológico, y a largo plazo, del estadio en que se intervino. Dado que la claudicación respiratoria es la complicación más frecuente, debe ponerse mayor atención a la valoración y preparación preoperatoria, así como al uso de catéter peridural para mejorar la analgesia e iniciar la terapia respiratoria más temprano. ${ }^{4,46}$ En 2 casos, a pesar de múltiples estudios que no lograron detectar recidiva tumoral, los pacientes fallecieron emaciados, posiblemente por intolerancia a su nueva situación digestiva.

La mortalidad quirúrgica del $10.7 \%$ y la supervivencia a 5 años del $31.5 \%$ aquí reportadas, no son estadísticamente significativas, porque se calcularon para los casos de resección curativa y paliativa y para todos los estadios juntos, además de que 5 pacientes que fallecieron durante el período, lo hicieron por otras causas. ${ }^{4,24,43,47}$

La calidad de vida de los sobrevivientes depende en gran parte de la manera en como acepte su nueva condición, pues van a tener algún grado de disfagia, deben dormir semiinclinados para evitar el reflujo, alimentarse frecuentemente con pocas cantidades y con alimentos adecuados, cuidar la dentadura y triturar los alimentos antes de deglutirlos. Cada vez que se apartan de lo indicado, se producen trastornos digestivos, disnea por llenura del tubo gástrico en el tórax, síndrome de dumping, etc. ${ }^{48}$

Se concluye que el cáncer del esófago, a pesar de que no representa una causa mayor de mortalidad en Costa Rica, se asocia con una baja supervivencia, debido al diagnóstico en estadio avanzado. A pesar del diagnóstico, que en los casos estudiados se realizó alrededor de los 3 meses de iniciada la disfagia, muchos pacientes tuvieron endoscopías previas por otros motivos y por lo menos en 8 existía el diagnóstico de mucosa de Barret asociada a síntomas de reflujo. ${ }^{18}$

Esto induce a recomendar que, pese a que no se justifican endoscopías masivas para detección de cáncer del esófago 
en Costa Rica, sí es necesario poner mayor atención al esófago cada vez que se realice una endoscopía por cualquier otra causa y a dar seguimiento a lesiones como la displasia, la esofagitis de reflujo y la presencia de mucosa de Barret. $^{49,50}$

El UTE debe llegar a ser un estudio de rutina y estar disponible en los hospitales mayores del Seguro Social.

El estudio y manejo de estos pacientes, así como la cirugía realizada por equipos con experiencia y dedicados, puede alcanzar resultados similares a los informados en la bibliografía, ${ }^{4,24,43,44,47,51}$ pero es preciso concentrarlos en pocos centros, en lugar de improvisar su tratamiento en forma anecdótica en todos los servicios y por muchos cirujanos.

\section{Abstract}

Between 1990 and 2006, 28 patients had esophageal cancer resection at the Department of Thoracic Surgery of the Hospital Calderón Guardia of the Costa Rican Social Security System. The mean age was 65 years with a high male predominance of $6: 1$. Eighty percent of the patients were smokers, $75 \%$ drinkers and 8 patients had previous diagnosis of Barret's esophagus. Dysphagia, epigastric pain and weight loss were the most frequent symptoms but the pain was related to preexisting peptic disease or esophagitis secondary to gastric reflux. Stage IIb, III and IV patients received neoadyuvant therapy. Three of these cases did not show residual tumor and 2 had residual aortic disease. Twenty one patients had total esophagectomy and 7 patiens had partial esophagectomies with gastric anastomosis in 5 and 2 esophagojejunostomies. Major complications were respiratory distress and bronchopneumonia in 7 patients, and anastomotic leak in 2 . Thirty days mortality was $10.7 \%$. The 3 patients died with bronconeumonía and one with cervical and other thoracic leak. Seven patients developed anastomotic strictures treated with dilatations. Survival at 5 and 10 years was $31.5 \%$ and $18 \%$ respectively.

\section{Referencias}

1. Registro Nacional de Tumores. Mortalidad por tumores malignos según localización anatómica. Costa Rica 2002.

2. Ide H, Tsutomu N, Nakamura T, Hayashi K, Endo T, Kobayashi A, Eguchi R, Hanyu F. Esophageal squamous cell carcinoma: pathology and prognosis. World J Surg 1994; 18: 321-330.

3. Becker HD. Esophageal cancer, early disease: diagnosis and current treatment. World J Surg 1994; 18:331-338.

4. Watson A. Operable esophageal cancer. Current results from the West. World J Surg. 1994; 18:361-366.

5. Enzinger PC, Mayer RJ. Esophageal cancer. N Engl J Med. 2003; 349:2241-2252.

6. Hölscher AH, Dittler HJ, Siewert JR. Staging of squamous esophageal cancer: accuracy and value. World J Surg. 1994; 18:312-320.
7. Van Dam J. Endosonographic evaluation of the patient with esophageal cancer. Chest. 1997; 112:184S-190S.

8. Lerut T, Coosemans W, Van Raemdonck D, Dillemans B, de Leyn P, Marnette JM, et al. Surgical treatment of Barrett's carcinoma: correlations between morphologic findings and prognosis. J Thorac Cardiov Surg. 1994; 107:1059-1066.

9. Daly JM, Fry WA, Little AG, Winchester DP, McKee RF, Stewart AK, Fremgen AM. Esophageal cancer: results of an American College of Surgeons patient care evaluation study. J Am Coll Surg. 2000; 190:562-573

10. Nishihira T, Nakamo T, Mori S. Adjuvant therapies for cancer of the thoracic esophagus. World J Surg. 1994; 18: 388-389.

11. Kelsen DP, Ginsberg R, Pajak TF, Sheahan DG, Gunderson L, Mortimer J, et al. Chemotherapy followed by surgery compared with surgery alone for localized esophageal cancer. N Engl J Med. 1998; 339:1979-1984.

12. Lerut TE, Leyn P, Coosemans W, Van Raemdonck D, Cuypers P, Van Cleynenbreughel B. Advanced esophageal carcinoma. World J Surg. 1994; 18: 379-387

13. Aikou T, Shimazu H. Difference in main lymphatic pathways from the lower esophagus and gastric cardia. Jpn J Surg. 1989; 19:290-295.

14. Altorki NK, Skinner DB, Segalin A, Stephens JK, Ferguson MK, Little AG. Indications for esophagectomy in nonmalignant Barrett's esophagus: a 10 year experience. Ann Thorac Surg. 1990; 48:724-727

15. Cameron AJ, Ott BJ, Payne WS. The incidence of adenocarcinoma in columnar-lined (Barret's) esophagus. N Engl J Med. 1985; 313:857.

16. Mainieri JA, Smith I, Mainieri G. Acalasia. Estudio de 27 casos en el Hospital Dr.Rafael Á. Calderón Guardia. Acta Médic Costarric. 2003; 45:25-30.

17. Gisbert JP, Losa C, Barreiro A, Pajares JM. Acalasia esofágica. Revisión de sus aspectos clínicos, diagnósticos y terapéuticos. Revista Clínica Española 2000; 200:424-431.

18. Bremner CG. Barrett's oesophagus. Br J Surg. 1989;76:995-996.

19. DeMeester SR, DeMeester TR. Columnar mucosa and intestinal metaplasia of the esophagus. Fifty years of controversy. Ann Surg. 2000; 231:303-321.

20. Lundell L, Olbe L. Current state of treatment of carcinoma of the oesophagus and gastro-oesophageal juntion. Clinical review. Acta Chir Scand. 1990; 156:193-202.

21. Shaheen N, Ransohoff DF. Gastroesophageal reflux, Barrett esophagus and esophageal cancer. JAMA. 2002; 287:1982-1986.

22. Rao DN, Sanghvi LD, Desai PB. Epidemiology of esophageal cancer. Sem Surg Oncol. 1989; 5:351-354.

23. Collard JM, Romagnoli R, Hermans BP, Malaise J. Radical esophageal resection for adenocarcinoma arising in Barrett's esophagus. Am J Surg. 1997; 174:3907-311.

24. Fok M, Law SYK, Wong J. Operable esophageal carcinoma: current results from Hong Kong. Worl J Surg. 1994; 18:355-360.

25. Swaroop VS, Damle SR, Advani SH, Desai PB. Nutrition and esophageal cancer. Sem Surg Oncol. 1989; 5:370-372.

26. Fok M, Cheng SWK, Wong J. Endosonography in patient selection for surgical treatment of esophageal carcinoma. World J Surg. 1992; 16:1098-1103.

27. Sharma OP, Subnani S. Role of computerized tomography imaging in staging oesophageal carcinoma. Sem Surg Oncol. 1989; 5:355-358.

28. Patel AN, Buenaventura PO. Current staging of esophageal carcinoma. Surg Clin N Am. 2005; 85:555-567.

29: Krasna MJ. Minimally invasive staging for esophageal cancer. Chest. 1997; 112:191S-194S.

30. Roth JA, Pass HI, Flanagan MM, Graeber GM, Rosemberg JC, Steinberg S. Randomized clinical trial of preoperative and postoperative adjuvant chemotherapy with cisplatin, vindesine and bleomycin for carcinoma of the esophagus. J Thorac Cardiovasc Surg. 1988; 96:242-248.

31. Forastiere AA, Heitmiller RF, Kleimberg L. Multimodality therapy for esophageal cancer. Chest. 1997; 112 195S-200S.

32. Gamliel Z, Krasna MJ, Multimodaly treatment of esophageal cancer. Surg Clin N Am. 2005; 85:621-630. 
33. Mariette C, Finzi L, Fabre S, Balon JM, Van Seuningen I, Triboulet J-PJ. Factors predictive of complete resection of operable esophageal cancer: a prospective study. Ann Thorac Surg. 2003; 75:1720-1726.

34. Hölscher AH, Siewert JR. Surgical treatment of early esophageal cancer. Dig Surg. 1997; 14:70-76.

35. Hölscher AH, Siewert JR. Surgical treatment of early esophageal cancer. Dig Surg. 1997; 14:70-76.

36. Nichols FC, Allen MS, Deschamps C, Cassivi SD, Pairolero PC. Ivor Lewis esophagogastrectomy: Surg Clin N Am. 2005; 85:583-592.

37. Horstmann O, Becker H, Nustede R, Verreet PR, Röher HD. Drainage of cervical esophagogastrostomy: essential or dangerous? Dig Surg. 1997; 14:88-91.

38. Wilson SE, Stone RS, Scully M, Ozeran L, Benfield JR. Modern management of anastomotic leak after esophagogastrostomy. Am J Surg. 1982; 144:95-101.

39. Iannettoni MD, Whyte RL, Orringer MB. Catastrophic complications of the cervical esophagogastric anastomosis. J Thorac Cardiovasc Surg. 1995; 110:1493-1501.

40. Soga J, Ohyama S, Suzuki T, Tanaka O, Sasaki K, Ishizaki E, Muto T. Carcinomas involving the esophagogastric juntion. Int Surg. 1990; 75:205-207.

41. Lin J, Iannettoni MD. Transhiatal esophagectomy. Surg Clin N Am. $2005 ; 85: 593-610$
42. Hennessy TPJ. Lymph node dissection. World J Surg. 1994; 18:367372.

43. Zhang DW, Cheng GY, Huang GJ, Zhang RG, Liu XY, Mao YS, Wang YG, et al. Operable squamous esophageal cancer: current results from the East. World J Surg. 1994; 18:347-354.

44. Chassin JL. Esophagogastrectomy: Data favoring end-to-side anastomosis. Ann Surg. 1978; 188:22-27.

45. Bardini R, Asolati M, Rulo A, Bonavina L, Baseggio S, Peracchia A. Anastomosis. World J Surg. 1994; 18:373-378.

46. Law SYK, Fok M, Wong J. Risk analysis in resection of squamous cell carcinoma of the esophagus. World J Surg. 1994; 18:339-346.

47. Altorki NK, Girardi L, Skinner DB. Squamous cell carcinoma of the esophagus: therapeutic dilemma. World J Surg. 1994; 18:308-311.

48. Gelfand GAJ, Finley RJ. Quality of life with carcinoma of the esophagus. World. J Surg. 1994; 18:399-405.

49. Ranson JM, Patel GK, Clift SA, Womble NE, Read RC. Extended and limited types of Barrett's esophagus in the adult. Ann Thor Surg. 1982; 33:19-27

50. Berenson MM, Riddell RH, Skinner DB. Malignat transformation of esophageal columnar epithelium. Cancer. 1978; 41:554-558.

51. Arce VJ, Fernández SA, Pucci CJJ, Urzola GE, Flores ME, Bolaños EM. Sustitución del esófago por el estómago sin toracotomía. Act Méd Costarric. 1979; 22:365-372.

\section{Costa Rica en el exterior}

\section{Simpatectomía toracoscópica para taquicardia ventricular polimorfa incesante en miocarditis chagásica crónica.}

Gutiérrez O., Garita E., Salazar C.

International Journal of Cardiology 2007, 119: 255-257

El manejo de las arritmias ventriculares complejas debidas a la miocarditis chagásica es un verdadero reto. Presentamos el caso de un hombre joven que se presentó con una taquicardia ventricular polimorfa bien tolerada de origen desconocido. El cuadro era resistente al manejo médico convencional y quien como último recurso fue sometido a una simpatectomía toracoscópica bilateral, permitiendo entonces un mucho menor manejo de su arritmia. Eventualmente los resultados de sus exámenes mostraron que tenía enfermedad de Chagas y falleció súbitamente 8 meses después.

Análisis de la estructura genética de tres poblaciones hispanas de Costa Rica, México y del suroeste de los Estados Unidos de América utilizando marcadores STR de cromosomas-Y y secuencias de ADNmt

Campos-Sánchez R, Barrantes R, Silva S, Escamilla M, Ontiveros A, Nicolini H, Mendoza R, Munoz R, Raventos H.

Centro de Investigación en Biología Celular y Molecular, Universidad de Costa Rica, San Pedro, SJ, Costa Rica, USA.

Hum Biol. 2006 Oct; 78 (5): 551-63

Se estudiaron doscientos diecisiete sujetos masculinos de Costa Rica, México y de la población hispana del suroeste de los Estados Unidos de América. Se analizaron doce secuencias STR del cromosoma Y y la secuencia HVSI del ADNmt para describir su estructura genética y para comparar sus linajes maternos y paternos. Todos los sujetos forman parte de dos estudios financiados por el NIMH para localizar los genes de susceptibilidad a la esquizofrenia en poblaciones hispanas con ancestros de México y Centroamérica. Mostramos que estas tres poblaciones son similares en sus características genéticas internas como lo revelan los análisis del ADNmt y de la diversidad del STR del cromosoma-Y. Estas poblaciones están relacionadas más fuertemente a través de su linaje materno que a través de su linaje paterno porque se observa un mayor número de haplotipos compartidos y polimorfismos en el ADNmt (en comparación con los STRs del cromosoma-Y). Estos resultados proveen evidencia de contactos previos entre las tres poblaciones e historias compartidas. Un análisis de la varianza molecular reveló que no hay diferenciación genética para el ADNmt en las tres poblaciones, pero se detectó diferenciación en los STRs del cromosoma Y. Análisis de la distancia genética mostró que las tres poblaciones están cercanamente relacionadas probablemente como resultado de migraciones entre vecinos cercanos como lo indican los haplotipos compartidos y las historias demográficas. Esta relación podría ser una característica importante para estudios genéticos en poblaciones latinoamericanas e hispanas. 